\title{
REFLEXIONES “CONTRACTUALES” ACERCA DE LA LEY DE INTERRUPCIÓN VOLUNTARIA DEL EMBARAZO EN CHILE
}

\author{
José Javier Caro Miranda ${ }^{1}$
}

Resumen: Después de un año de entrada en vigencia de la Ley de Interrupción Voluntaria del Embarazo en Chile, hoy se reconoce la posibilidad de abortar en tres situaciones específicas y se da apoyo profesional a las mujeres que la invocan. Se plantean reflexiones acerca de su aplicación en las causales descritas en ella. Se sitúa la dignidad humana como eje central de la interpretación de la realidad feto-materna. Se comenta el sentido del acto médico-perinatal, en el contexto de la preservación de la especie humana. Se explica la aplicación del principio del doble efecto y el del único bien posible en el discernimiento de la primera causal. En la segunda causal se comenta la influencia de la tecnología en la anamorfosis fetal, junto con la vulnerabilidad y la focalización autorreferencial materna en el feto malformado. En la tercera causal se intenta aplicar la noción de cómo la violencia no puede justificarse a si misma y se necesita mediar consensos para no enfocar al feto como causante del daño. Se pretende alertar sobre el riesgo de alienación de los profesionales de la salud ante el significado de la dignidad del ser humano.

Palabras clave: aborto voluntario, dignidad humana, inviabilidad fetal, violencia sexual, violencia fetal, morbilidad materna severa, anamorfosis

\section{“Contractual” reflections on Chile's law on voluntary pregnancy termination}

Abstract: After one year of the Law of Voluntary Interruption of Pregnancy in Chile, the possibility of aborting in three specific situations is recognized and professional support is given to the women who invoke it. Reflections are raised about its application in the causes described in it. Human dignity is placed as the central axis of the interpretation of the fetusmaternal reality. The meaning of the medical-perinatal act is discussed in the context of the preservation of the human species. The application of the principle of double effect and that of the only possible good in the discernment of the first causal is explained. In the second case, the influence of technology in fetal anamorphosis is discussed, together with the vulnerability and self-referential maternal focus in the malformed fetus. In the third case, we try to apply the notion of how violence cannot justify itself and we need to mediate consensus so as not to focus on the fetus as the cause of the harm. The aim is to warn about the risk of alienation of health professionals before the meaning of the dignity of the human being.

Keywords: voluntary abortion, human dignity, fetal inviability, sexual violence, fetal violence, severe maternal morbidity, anamorphosis

\section{Reflexóes “contratuais” acerca da lei de interrupçáo voluntária da gravidez no Chile}

Resumo: Depois de um ano da entrada em vigor da Lei de Interrupção Voluntária da Gravidez no Chile, hoje se reconhece a possibilidade de abortar em três situaçôes específicas e se dá apoio profissional às mulheres que a invocam. Propóem-se reflexốes sobre sua aplicação nas causas descritas nela. Situa-se a dignidade humana como eixo central da interpretaçáo da realidade fetomaterna. Comenta-se o sentido do ato médico perinatal no contexto da preservaçáo da espécie humana. Explica-se a aplicação do princípio do duplo efeito e o do único bem possível no discernimento da primeira causa. Na segunda causa comenta-se a influência da tecnologia na anamorfose fetal, junto com a vulnerabilidade e a focalização auto-referencial materna no feto mal formado. Na terceira causa tenta-se aplicar a noção de cómo a violência não pode justificar a si mesma e necessita buscar consensos para não colocar o feto como fator causador do dano. Pretende-se alertar sobre o risco de alienação dos profissionais da saúde diante do signififcado da dignidade do ser humano.

Palavras chave: aborto voluntário, dignidade humana, inviabilidade fetal, violência sexual, violência fetal, morbilidade materna grave, anamorfose

\footnotetext{
${ }^{1}$ Servicio de Obstetricia y Ginecología, Hospital Puerto Montt. Departamento de Obstetricia y Ginecología, Escuela de Medicina, Facultad de Medicina, sede Patagonia, Universidad San Sebastián, Puerto Montt, Chile

Correspondencia: pepecaro@gmail.com
} 


\section{Introducción}

La entrada en vigencia de la ley de "Interrupción Voluntaria del Embarazo" repuso la figura legal del aborto terapéutico en Chile, la cual ya existía en nuestra legislación desde 1931, hasta que en 1989 la dictadura militar lo prohibió en el artículo 119 del código sanitario. El marco jurídico que la sustenta hace referencia a la Declaración Universal de los Derechos Humanos en cuanto a que "todos los seres humanos nacen libres e iguales en dignidad y derechos" en el contexto de las garantías de libertad e igualdad entre mujeres y hombres que un Estado debe ofrecer respecto de la igualdad de género y los derechos reproductivos de la mujer. Así se configuran tres causas de interrupción del embarazo: 1) cuando existe riesgo vital de la madre durante la gestación; 2) cuando el feto presenta una condición que es incompatible con la vida extrauterina, y 3 ) en el caso de éste sea el producto de una violación ${ }^{2}$.

El 10 de febrero de 2018 se realizó la primera interrupción por la tercera causal en Chile a una niña de 12 años procedente de Chiloé. El procedimiento se realizó en la Santiago, porque el centro de salud en el que se atendía presentó objeciones. El caso era delicado, complejo y dramático. El diario en línea The Clinic se refiere a este evento como "un pequeño paso para la medicina chilena y un gran paso para todo el país"3.

La normativa llegó a todos los servicios de salud del país y sus hospitales. En el sistema público se han constituido equipos multidisciplinarios (psiquiatra, psicólogo, trabajadora social, obstetra), respetando la objeción de conciencia de los médicos, y se cuenta con el arsenal terapéutico para ofrecer el procedimiento de la interrupción en un proceso de consejería y acompańamiento, propiciando el principio bioético de autonomía de la embarazada para decidir si continúa o no con la gestación. Hoy está en discusión si las instituciones privadas pueden presentar objeción de conciencia.

Durante 2018 se han realizado 528 interrupciones voluntarias del embarazo bajo la tutela de esta ley, de las cuales el 45,9\%, 37,6\% y $16,5 \%$ co-

${ }^{2}$ Disponibleenhttps://www.leychile.cl/Navegar?idNorma $=1108237$

${ }^{3}$ Disponible en http://www.thedinic.d/2018/02/10/primer-aborto-legal-chile/ rrespondieron a la causal uno, dos y tres respectivamente ${ }^{4}$.

Como puede observarse esta no es una ley de aborto libre, en la que el Estado permita a la mujer decidir (autodeterminación) sobre la continuidad de la gestación, en el ámbito de su deseo de control de la natalidad. Está orientada a un grupo de mujeres que presentan problemas en o por la gestación y a las víctimas de violencia sexual.

Antes de presentar algunos tópicos para reflexionar lo que esta ley nos presenta, hay que aclarar que la interrupción del embarazo puede ocurrir a cualquier edad gestacional y no necesariamente constituir un aborto. En la obstetricia existen patologías propias del embarazo que afectan al binomio madre-hijo, que obligan a interrumpir la gestación y que no son sometidas al escrutinio de la ley. Lo que importa es la viabilidad fetal; de hecho, en casos graves con daño severo, la interrupción de embarazos con edad gestacional menor a 28 semanas representa un manejo de mucha complejidad perinatal, con elevada morbimortalidad neonatal y con secuelas importantes en la vida posterior del recién nacido. Por esta razón, los equipos de salud perinatal concentran sus esfuerzos en lograr la mayor edad gestacional posible. El límite de la viabilidad en la mayoría de los centros hospitalarios de Chile está en las 25 $\operatorname{semanas}^{5}(1)$, bajo esa edad gestacional la sobrevida es anecdótica. Por lo tanto, se entiende que, ante la interrupción por una enfermedad materna grave concurrente en el embarazo, o preexistente y agravada por éste, todos los equipos harán sus mejores esfuerzos terapéuticos, según el estado del arte de la medicina para prolongar la gestación más allá de las 25 semanas y, ante la interrupción inminente, preparar lo mejor posible al feto para su vida extrauterina en un centro ad hoc (con intervenciones perinatales efectivas según la medicina basada en evidencia).

La definición de aborto es "evitar el nacimiento" (del latín ab: evitar, privar y ortus: nacer). Puede ser provocado por terceros o espontáneo, es decir, ocurre en su propio devenir por diferentes causas. En estricto sentido, el acto de nacer de un

\footnotetext{
${ }^{4}$ Disponible en http://www.minsal.cl/live-reporte-mensual-actualizado/

${ }^{5}$ Disponible en http:/www.deis.cl/wp-content/uploads/2011/05/ SerieNacimientos_2000-2015.html
} 
feto vivo tiene dos efectos; uno es el salir del lugar de gestación, que es el útero, y el otro de haberlo hecho para continuar viviendo. En el caso del feto muerto, solo corresponde el primer efecto. La definición operacional de "aborto" implica la no viabilidad al momento de nacer (vivo o muerto), la que está establecida hasta las 22 semanas de gestación; es decir, la imposibilidad de mantener cualquier función vital que permita la existencia por la inmadurez biológica del que nace. La connotación mas compleja la tiene el que es provocado, porque implica evitar que el embarazo llegue a un punto de viabilidad neonatal y, por lo tanto, que prosiga su existencia. Así se da por entendido que existe el nacer en un rango de normalidad de tiempo entre las 37 y 41 semanas de gestación (término), uno que no es normal entre las 22 y 36 semanas (prematuro o pretérmino) y cuando es menor de 22 semanas (aborto).

Las causales de interrupción número uno y dos pueden estar presentes en cualquier momento del curso del embarazo, desde el principio hasta el final, pasando por las definiciones descritas de aborto, prematuridad y parto de término.

La causal número tres implica la definición de aborto propiamente tal (para que ese ser no exista), porque lo demás sería alcanzar la viabilidad lo más pronto posible para entregar el feto/neonato a otro cuidador. Puede ser invocada hasta las 14 semanas de gestación por amenorrea.

Sin embargo, este problema multidimensional necesita ser meditado desde un ámbito bioético, filosófico, antropológico y sociológico. El objetivo es presentar algunas reflexiones acerca del contexto del aborto en Chile en cuanto a qué significa el acto médico perinatal, las implicancias ético-filosóficas de cada una de las indicaciones de la Ley IVE, y el peligro de alienación profesional ante el sentido de la dignidad humana.

\section{Desde la negación hacia el reconocimiento de un conflicto}

La ley actual adolece de cierta extemporaneidad, porque es una respuesta tardía a aquellas muertes ocurridas por esta causa antes de la década de los noventa, ya que el aborto era la primera causa de defunción materna. Hoy la situación es distinta, porque Chile está entre los tres países con menor mortalidad materna en América ${ }^{6}(2)$.

Una democracia no puede tener sostenibilidad sin enfrentar los conflictos originados por los problemas reales que sus ciudadanos viven y el aborto es uno de ellos. Según Bauman, un punto débil de las democracias es "la contradicción entre la universalidad formal de los derechos democráticos y la no tan universal capacidad de sus titulares para ejercerlos con eficacia" (3). Se abre así una brecha en la cual las personas deben resolver sus problemas como puedan y por sus propios medios. Eso es lo que ocurría en el Chile de hace 50 años, con una alta tasa de muertes maternas por aborto ilegal y provocado como método de control de la natalidad. Por esta razón, un Estado "social" debe ocuparse del tema, legislando y educando. Eso es lo que está ocurriendo hoy día, por lo que el compartir y debatir ideas abre el camino de las soluciones. La despenalización del aborto es una forma de representar el concepto de equidad (justicia social) (4) entre los ciudadanos, respecto de la consecución del mejor desarrollo humano que se pueda alcanzar. Toda inequidad es una forma de diferencia injusta, por lo tanto, el aborto penalizado y no reconocido se convierte en un evento que la gatilla, cuando los ciudadanos tienen acceso (al margen de la ley) a realizar un aborto en una gradiente diferencial de calidad y seguridad personal, según el nivel socioeconómico al que pertenecen y los recursos monetarios que disponen.

El respeto por las opciones que toman los individuos frente a sus problemas vitales, con información pertinente y oportuna, se garantiza con educación respecto de estos temas fundamentales, por lo que el Estado necesita promover y generar políticas públicas de educación, salud y de justicia respecto del aborto, que sean reflexionadas no solo desde las necesidades individuales y colectivas de desarrollo humano, sino también desde el ideario de sociedad que se quiere alcanzar.

En la era del individuo posmoderno, las elecciones se basan en el hiperconsumo transitorio, seductivo y diferenciado que se caracteriza por la "de-sacralización, des-trascendentalización e insustancialización continua de lo humano" (5). Por

\footnotetext{
${ }^{6}$ Fundación Gapminder. Disponible en https://www.gapminder. org/tools/
} 
lo tanto, es necesario primero lograr un punto de equilibrio entre el sentido de la dignidad humana y la libertad individual. Según Simone Weil, la libertad no está definida por la relación entre deseo y la satisfacción, sino por el pensamiento y la acción(6); dicho de otro modo, la reflexión acerca de las necesidades del ser humano para disponer por sí mismo y la voluntad de hacer algo respecto de ellas. Aquí el valor que otorgamos a la cualidad de ser humano está definido por la concepción de dignidad como elemento de referencia para cualquier problema relacionado con el ser en el tiempo y espacio.

\section{Reflexionar el acto médico obstétrico}

Toda acción en el mundo natural "inaugura una realidad nueva sin precedentes ni en la consciencia ni en el deseo, algo nuevo en la propia biografía y algo nuevo en el acontecer del mundo". En el hacer se revela el significado de la acción del sujeto que la realiza(7). En este punto, el acto médico de la atención obstétrica perinatal se enfrenta a dos sujetos de relación: la madre y el feto. Cuando interactuamos con el Otro se genera el problema moral de nuestros actos. Lo relativo a la objeción de conciencia surge de esta tensión entre el significado y el sentido del acto que se realiza desde el sujeto, que es el operador médico, hacia el Otro (otros). El médico ve y aprecia en el periodo de gestación humana a dos pacientes, su paradigma de formación es alcanzar el máximo potencial de salud/vida para ambos a lo largo del proceso del embarazo. Podemos inferir que el significado de la acción médica prenatal es proteger, mantener y promover la salud de la madre y el feto, y su sentido se revela en la protección y preservación de la especie humana. El aborto despenalizado nos coloca en la disyuntiva de aceptar realizar un acto con el que estamos o no de acuerdo; en este contexto, aparece el deber ser para el médico (preservar la salud/vida de la madre y la del feto), evaluando y decidiendo si el acto sirve o no para conseguir algún bien según los valores en los que la acción médica tiene significado y sentido. Y, por otro lado, está el respeto por la autonomía y autodeterminación de las personas; en este caso, la mujer con respecto a su propio embarazo. Como dice Millas: "en el prójimo reconocemos intuitivamente a un ser idéntico a nosotros mismos..., el para sí individual se expande de este modo más allá de sí mismo, bajo la forma del alter ego, del otro yo, en quien se reproduce el hecho y la dignidad del ser hombre que conocemos como referencia propia" (8). No solo lo hacemos físicamente, "lo que experimentamos es la intimidad del otro, paradojalmente, como una intimidad inviolable - no experimentada, diríamos-, semejante a la mía" (9), la existencia ajena la percibimos como un hecho de valor. He aquí lo medular de la objeción de conciencia para ser parte del proceso del aborto legalizado, que algunos médicos han hecho notar. La intención moral reclama ser instrumentalizada por el derecho positivo, para que este conflicto ético - entre la acción y la intersubjetividad - pueda ser un derecho concreto de la mujer autodeterminada (informada, empoderada, consciente,) y que el médico (el sujeto que realiza la acción del aborto) no sea imputable moral ni legalmente. Sin embargo, el operador observa, entiende y califica el conflicto de intereses y valores, para decidir la propia y subjetiva imputabilidad moral de sus actos.

\section{Vencer la entropía}

La definición de cuándo comienza la vida es arbitraria y, más aún, lo que consideramos un ser humano. La biología nos permite entender el proceso de la fecundación y cómo se inicia un linaje celular distinto al de sus progenitores. La inmanencia del alma en el inicio de la vida, como concepto metafísico, no la conocemos, solo sabemos acerca de la organicidad de un proceso que, incluso hoy, se puede manipular con precisión en parejas con problemas de fertilidad. Este problema de definiciones será siempre arbitrario: no hay consenso respecto de ese momento primero que llamaremos "inicio de la vida" y por ende "existencia". Pero intuimos al ser humano en estas etapas precoces. La definición jurídica de "persona" es arbitraria y en las etapas iniciales del ser humano no tiene límites precisos.

Para Teilhard de Chardin "La vida es, contrariamente al juego nivelador de la entropía, la construcción metódica, sin cesar engrandecida, de un edificio cada vez más improbable. El Protozoo, el Metazoo, el ser sociable, el Hombre, la Humanidad... son otros tantos desafíos crecientes a la entropía" (10). La entropía funciona como un obstáculo frente a la vida molecular y celular, que 
necesita cantidades ingentes de energía y, aun si así marchamos hacia lo improbable, acaso la vida humana ¿no ha demostrado ser una creación infalible en su diseńo y que no se agota en su devenir? $\mathrm{Y}$, bajo esta mirada, especialmente aquellos que ejercemos la medicina perinatal, podemos colegir y hacernos una idea de dónde comienza la vida y, con ella, los orígenes del sentido de lo humano, en seres "con teleonomía, morfogénesis autónoma e invarianza reproductiva" (11).

\section{Primera causal. Principio del doble efecto y único bien posible}

En el mundo médico, las consideraciones finales de esta causal son cuestionadas a priori solo para definir con máxima precisión la probabilidad de que sobrevenga un evento adverso para la madre embarazada, es decir, es un análisis con enfoque bayesiano, en el que se valora la probabilidad del evento muerte o dańo materno severo. En el estado actual del arte en la medicina, son escasas las enfermedades maternas que justifican esta causal y los equipos médicos tienen herramientas éticas cuando se enfrentan una situación de éstas.

Según Protágoras, "Homo omnium rerum mensura est" (El hombre es la medida de todas las cosas). De aquí podemos colegir que la medida del valor de la vida del ser humano es la del otro. En la primera causal, cuando la vida de la madre está en peligro, en iguales condiciones de dignidad del ser humano, se opta por la interrupción de la gestación para salvar la madre sin desconocer ni negar la dignidad y el valor del feto. Si él es abortado o muere, éste no era el fin perseguido pues la cadena de eventos ya ha determinado que ese feto será expulsado del útero o dejará de existir (ley/doctrina/ principio del doble efecto). Valenzuela(12) define con precisión hermeneuta las situaciones clínicas en que su aplicación es correcta y adecuada, como en los casos de aborto séptico, cáncer de cuello uterino, enfermedad trofoblástica y embarazo ectópico complicado, todas ellas patologías que, de no intervenirse a tiempo, pueden comprometer gravemente la vida de la embarazada. En estas circunstancias, la no imputabilidad de un efecto secundario de una acción está condicionada por: "1) la acción, el objetivo y la intención deben ser buenos, no malos o neutros (indiferentes), 2) la acción directamente no intenta mal alguno, 3) el efecto bueno no es consecuencia del mal, este no es un medio para conseguir un bien, y 4) el resultado bueno debe ser mayor (mejor) que el efecto malo (proporcionalidad)" (12).

Sin embargo, la reflexión acerca de este principio se hace más compleja, pues hay casos en que el feto, directa o indirectamente, compromete la salud de la madre, ya sea por sus requerimientos metabólicos o factores endógenos placentarios/ fetales, que llevan a una alteración patológica terminal de la adaptación materna al embarazo (v.gr. pre-eclampsia severa, síndrome HELLP, hígado graso agudo hipertensión pulmonar materna primaria, cirrosis biliar materna). Es decir, es el feto la causa de morbilidad materna grave y muerte materna consiguiente, por lo tanto, su remoción es la solución, lo que coincide con matar al feto. El efecto primario que se busca es remover la causa de la potencial muerte de la madre (eliminar el feto), "bueno" para la madre y "malo" para el feto. El resultado alternativo a la realización del acto de abortar es su omisión, lo que implica que el feto morirá de todas formas al morir la madre. De tal forma, eliminando y/o matando al feto se logra al menos salvar la vida de la madre. Por lo tanto, no corresponde a la aplicación del principio del doble efecto. Se aplica más al principio del mal menor, o, en un sentido positivo, el máximo bien posible (en este caso el único bien)(12).

\section{Segunda causal. Tecnología, anamorfosis y vul- nerabilidad}

La ciencia ha logrado un nivel de conocimiento y entendimiento de la naturaleza humana que jamás habíamos soñado. Para la medicina actual, este es el argumento que ayuda a tomar decisiones con objetividad. La ciencia avanza en lo desconocido, develando parciamente los fenómenos que estudia, ayuda a descubrir cómo opera el ser, pero no lo explica: se necesita de una metarreflexión para construir y descubrir el sentido del ser humano con sabiduría $(13,14)$. La medicina, por lo tanto, desarrolla su quehacer en este ambiente científico de carácter histórico-epistémico con un sentido ontológico, generando la necesidad una reflexión que va más allá de la ciencia (acto de filosofar), este es el arte de la medicina. Sin embargo, este arte ha sido desplazado, como en muchos ámbitos de la vida humana, por la técnica y la 
tecnología (con función explicativa y descriptiva a corto plazo), que nos llenan de información y que muestran lo oculto a nuestros ojos en un contexto de mecanización, que es "intelección y, como el intelecto es principalmente utilitario, no hay esteticismo espiritual ni espiritualidad ética en la máquina” $(15,16)$. Es decir, el acto médico, que es profundamente humano, involucrando a un ser con otro con un sentido de entrega bilateral, se confronta con una tecnología ausente de involucramiento, que se agota en su focalización y especificidad, entregando información que a veces resulta inconmensurable e ininteligible por quien la recibe (y para quien la recaba), que toma distancia frente a la naturaleza del hecho y se desentiende explicando "eso" y nada más, fragmentado, sin unicidad(15). "Más información o una acumulación de información por sí sola no constituye ninguna verdad, no elimina la fundamental imprecisión del todo. Más información no conduce de manera necesaria a mejores decisiones"(16). Esta reflexión es válida para el uso de la ultrasonografía en la obstetricia, con todas sus técnicas de observación y medición en la predicción de alteraciones cromosómicas, en la visualización directa de malformaciones o anomalías anatómicas estructurales del feto, las que pueden confluir en una miríada de síndromes y enfermedades, con pronósticos de mortalidad perinatal muy disimiles. También, las técnicas genéticas, muy complejas y onerosas, con un nivel de precisión y valores predictivos elevados. Con estas herramientas se puede predecir y pronosticar, pero siempre con un margen de error. La interpretación de los resultados, la visualización del contexto, la relación médico-paciente con la embarazada, las habilidades blandas para entender lo que ella pide o solicita, sus miedos, sus esperanzas; todo configura un acto médico complejo, para aconsejar sin sesgo acerca de lo que significa el hallazgo realizado, tanto para la embarazada como para el feto, que es quien tiene la enfermedad (defecto o patología) y las alternativas que existen. Entender y asumir el significado de lo que se ha encontrado, relativo a lo digno para la madre y el feto, es decir, a ser merecedor de ese estar existiendo ahora y con su fin último potencial, es el objetivo de la relación médico/binomio madre-hijo(a). Definir la incompatibilidad con la vida no es un proceso sencillo, es probabilístico e indefinido, pues jamás sabremos con precisión y exactitud cuándo ocurrirá. Por lo tanto, es factible cuestionar la certeza del momento en que ocurrirá el cese de las funciones vitales del feto con anomalías estructurales/genéticas, tanto durante la gestación como después de nacer.

"Usar adecuadamente el lenguaje permite acercarse a las cosas con discreción, atención y cautela, con respeto hacia aquello que las cosas comunican sin palabras"(17). La imagen ecográfica bidimensional o tridimensional habla por sí sola sobre la morfología del feto a los ojos de un examinador entrenado, no así para la embarazada y su familia. El examinador ecográfico asume que la descripción es suficiente para que se entienda lo que sucede, pero olvida que, en el imaginario parental, cualquier anomalía es sinónimo de un proceso de anamorfótico, en el que "la composición de dos perspectivas no es integrable en una perspectiva general", desvirtuando el sentido morfológico del embarazo focalizándose solo en la forma y la imagen $(18,19)$. No solo lo que se dice, sino toda gestualidad, todo silencio comunican algo incómodo, terrible, feo. Se debe tener discreción y cautela respecto de lo que se dice y lo que se calla, de cómo se comunica lo observado. Una anomalía fetal estructural no es una monstruosidad, es una enfermedad que nos sitúa en un espectro de opciones, siendo la peor el cese de la vida.

Enfermar durante cualquier momento del ciclo vital humano es una situación altamente probable desde la gestación, situando al ser humano en un escenario de vulnerabilidad permanente. La pregunta que surge es si enfermar, tener una discapacidad, una anomalía, anormalidad o como quiera llamársele, ¿es una causa para negar el derecho a continuar viviendo?, en el caso del feto, intrauterinamente y, en el caso del que nace, por lo que resta de su existencia, aunque sean minutos, horas o días. ¿No es acaso en este estado de vulnerabilidad humana, donde ocurre la conexión que logra que toda subjetividad opere más allá de sí misma, en que el hombre reconoce en la alteridad del Otro eso que lo transforma en un ser finito?(20). Al distinguir esa fisura de la existencia surge la conciencia de la muerte, la finitud de la vida propia y, a la vez, la trascendencia, lo infinito de la vida a través de la existencia de otros y no solo la de su propia naturaleza. Si es así, al reco- 
nocer la dignidad y la vulnerabilidad del Otro con un sentido trascendente, ¿cuál es el objetivo de apurar su no existir, su no trascender, su no vivir por el tiempo que tenga? Es decir, cada vez que la vulnerabilidad del ser humano alcance un grado cualificado como severo ¿es mejor no existir, dejar de hacerlo?

La compasión aparece con la vulnerabilidad y el sufrimiento. Aparecen los conceptos como el de "morir bien", "acompañar en el morir", respeto por los ciclos vitales, por no intervenir acelerando su final. Es pertinente cuestionarnos si abreviar los procesos mengua el sufrimiento y el dolor, si lo hace desaparecer o si se olvida más rápido. ¿Y por quién hacerlo? ¿Solo por los padres y familiares? ¿Por la comunidad organizada? ¿Y qué pasa con el feto que debe sufrir el proceso de la intervención intrauterina para el aborto con la supresión hormonal, pasando por la inducción farmacológica de contracciones uterinas hasta la evacuación del contenido uterino con instrumental quirúrgico? ¿Acaso no siente dolor? ¿¿No sufre estrés biológico? Desde el punto de vista de adaptación materna al embarazo, muchos de los fenómenos que aparecen en la gestación son comandados por la unidad feto-placentaria, como por ejemplo los cambios en el metabolismo materno para tener mayor disponibilidad de glucosa para el feto, o el inicio del trabajo de parto con producción de hormonas por parte del feto. Y si bien estos eventos - que explicados desde el punto de vista biológico están en el contexto evolutivo de nuestra especie - tienen un estricto sentido teleológico para el ser humano en gestación, sin ellos no llegaría a concretarse su estar existiendo, ahora ni en el futuro, aunque aún no exista conciencia y/o subjetividad tal y como la percibimos.

La finitud de la vida, expresada en la letalidad que puede tener una determinada enfermedad, no es un argumento para acelerar el proceso vital, pues niega toda posibilidad de expresión de dignidad en el ser humano que lo sufre y cuya vulnerabilidad, en este caso, está expresada en una dependencia absoluta de su madre.

La salud, en su contribución al bienestar humano, tanto individual como colectivo, es un estado dinámico que expresa y a la vez permite el "ser uno mismo", permite la vida más allá de la vi- talidad de la biología y la mera sobrevivencia, es el punto de equilibrio de muchos determinantes que influyen en la vida de todo ser. Si la salud materna se focaliza en una actitud con sentido inmediato y autorreferencial respecto al feto enfermo - letalmente enfermo-, en que solo importa la malformación y la autodeterminación de la madre para acelerar el proceso por el cual sobrevendrá la muerte del feto, durante o después de la gestación, sin considerar la autopoiesis biológica y autodeterminación óntica de éste, es quitarle todo valor humano, es denegar su alteridad con su propio existir-devenir intrauterino, configurando un acto materno de ribetes narcisistas, en el que el feto representa únicamente el sufrimiento de la madre y no su propio sufrir, dolor o incertidumbre vital(21). Es una forma de ausencia de vínculo en que no hay encuentro con el Otro, se trata aquí de anular toda la experiencia. "En las experiencias se da el encuentro con el otro. Estas son alter-adoras. Las vivencias, en cambio, suponen una extensión del yo en el otro, en el mundo. Son pues, igual-adoras... Lo propio se emplaza frente al otro. Se sostiene en su alejamiento del otro... Si se pierde la relación con el otro, no se puede constituir una imagen sólida del yo"(21).

\section{Tercera causal. Violencia vs. violencia}

Esta causal es la más difícil y compleja de ponderar, por la circunstancia deshumanizada en que ocurre la gestación y que constituye un delito. El rechazo hacia el embarazo se explica, en este contexto, por la ausencia de consentimiento para gestar una nueva vida y por la violencia del acto en sí. Este quiebre de la voluntad de la persona puede ser el argumento para justificar el aborto.

El Estado se hace cargo de esta situación de vida límite de la mujer con la ley IVE, despenalizando el aborto hasta cierta edad gestacional, como una forma de apoyo a las víctimas, para que puedan tomar la decisión libremente. La gestación después de una violación puede ser interrumpida para que la persona agredida no se haga cargo, física y emocionalmente, de ese potencial ser humano. No hay contradicción con no desear ser la portadora de un embarazo no planificado ni deseado, producto de un acto violento, y optar por la interrupción de la gestación, porque esta puede hacerse para entregar un recién nacido a otro cui- 
dador (familia o Estado), manteniendo su dignidad como ser humano. El contrasentido aparece al optar por el aborto, negando el existir de ese ser que ya esta haciéndolo, a pesar del contexto de atrocidad y vulneración en que ha ocurrido la concepción, pues no es él quien ha producido el daño y se castiga el acto de la violación con la negación de su dignidad y existencia y no al que ha perpetrado el daño (el violador). Es decir, el acto de abortar se transforma en un acto que ejerce violencia contra un ser humano vulnerable, para proteger el bienestar de otro ser humano vulnerado. Este conflicto de intereses (madre vs. feto) no es dirimible sin antes haber analizado el operar de este contrasentido, y de tratar de alcanzar la máxima comprensión del fenómeno para realizar la ponderación moral que corresponde acerca de dónde está el mayor daño para la madre y si éste será evitado al realizar el aborto.

Una alternativa que se abre, después de un análisis objetivo de cada caso en esta causal y de no enfocar la provocación del daño en el nasciturus, es cuantificar el daño en la madre y evaluar el riesgo vital de ella (reflejado en un índice de calidad de vida futura), para interpretar el caso particular en el contexto de la primera causal. Corresponde ciertamente una ética de los consensos o situacional, con la esperanza de que las víctimas afectadas logren una percepción de gradualidad o proporcionalidad para considerar la dignidad del otro que se gesta en esta causal, que es de resultado todo o nada.

De alguna forma, debe debatirse si la violencia se alivia con violencia. Las leyes regulan el poder/ fuerza con que se ejerce el castigo, lo modulan en su intensidad, gradúan la respuesta de la sociedad organizada sobre un sujeto que ha sido juzgado y hallado responsable. En el caso de la tercera causal, una vez ocurrido el embarazo, ¿¿donde focalizar el objetivo de la legislación si no hay culpable al que castigar? ¿Si solo hay inocentes a los que proteger? “¿Cómo se integra la violencia en la estructura del mundo racionalmente concebido y valorado por el hombre? ¿Cómo se funda ella misma o qué cosas fundamenta, si fundamenta alguna?"(22). El feto, en este caso, será víctima de una violencia peculiar, no simplemente la fuerza con la que será interrumpida su existencia, sino una en que no hay apelación para la víctima, es una violencia que se institucionaliza en un sistema normativo que pasa inadvertido frente a otros humanos, que perciben la crueldad y brutalidad de una violación, pero no pueden o no quieren valorar lo que pasa con el feto. Si se trata de seguridad jurídica, tal como tratamos de calibrar los instrumentos de medición en las ciencias médicas, respecto del valor del Derecho y del respeto por la vida al menos aparece una contradicción difícil de medir. Dejarnos llevar por una idea de justicia y equidad, en que la madre decide sobre su embarazo (sobre un tercero), en esta causal, por el rechazo que todo ser humano manifiesta frente a la violencia, encubre u oscurece una realidad axiológica y se transforma en una falacia filosófica.

\section{Conclusiones}

La ley fundamenta su accionar en un marco teórico carente de estas reflexiones, y no ha tomado en cuenta las consideraciones ontológicas del que será abortado o que nacerá prematuramente y que, a fin de cuentas, son las mismas de aquel que continuará su gestación. Nos preocupa el alcance conceptual del diseño de esta ley que, buscando ser justa y basada en garantizar la libertad individual de una persona que comparte el mismo bien del feto, que es la vida, fundamente una lógica que, utilizada e interpretada en otros contextos del ciclo vital del ser humano, justifique el mismo acto, némesis de un valor distorsionado generando así una sociedad distópica. Cuando los acontecimientos son justificados, explicados y resueltos ya no son contingentes, dejan de importar y se convierten en una interpretación normal de la realidad. Así, la ley y la norma se transforman en un sistema de coacción intelectual, en el que las reglas son asumidas sin cuestionar lo fundamental(23), haciendo que los profesionales de la salud corran el riesgo de alienación ante el significado y la dignidad del ser humano, alejándonos tanto de la dialéctica del conflicto como de la hermenéutica de su significado. Y si bien actuamos en concordancia con los postulados de la bioética respecto a la mujer embarazada, en el caso del feto enfermo o gestado en una violación se estaría transgrediendo los principios de no-maleficiencia y de justicia, al usar un patrón de estatus de ser humano distinto entre ambos. 
Consideramos importante alertar a la comunidad médica sobre una ley cuyas bases no han considerado la dignidad humana del feto como elemento central de análisis en la solución del problema. Sentimos la obligación de hacer este "comentario", de "interrogar al discurso sobre lo que este dice y ha querido decir, tratar de hacer surgir... lo que jamás ha sido pronunciado"(24). No cuestionarnos acerca del valor de la dignidad humana es la fisura que debilita un postulado básico de Kant en el Derecho, pues todo y cada ser finito tiene dignidad (valor) y puede expresar su arbitrio sobre las cosas sensibles de este mundo(25) (lex iuris). El ser es el ser humano, especie que no se agota en su esencia y que siempre descubre nuevos horizontes ontológicos que expanden su significado y valor en este universo(9). La historia está repleta de episodios en que la vida del ser humano ha sido vulnerada solo por aceptar y asumir que existen categorías distintas de dignidad entre ellos.
Por último, el debate sobre aborto terapéutico es cuestión de todos los chilenos y no solo de un género. Sustentar su legalidad en consideraciones de género y violencia es completamente válido y pertinente, pero hace falta ampliar las visiones y puntos de análisis crítico que sirven de base para la construcción de una mejor comprensión entre aquellos cuyos principios y convicciones respecto de algo son distintos. 
Reflexiones "contractuales" acerca de la ley de interrupción voluntaria del embarazo en Chile - José Javier Caro Miranda

\section{Referencias}

1. Williams. Obstetricia. McGRAW-HILL e Interamericana editores, 23a edición; 2011: 215-226.

2. Koch E. Epidemiología del aborto y su prevención en Chile. Revista Chilena de Obstetricia y Ginecología 2014; 79(5), 351-360. DOI: https://dx.doi.org/10.4067/S0717-75262014000500001

3. Bauman Z. Daños colaterales. Desigualdades sociales en la era global. Fondo de Cultura Económica; 2011: 23.

4. Donoso Sabando C. Despenalización del aborto en Chile. Una cuestión de justica social. Acta Bioethica 2016; 22(2): 159-167.

5. Charles S, Lipovetsky G. Los tiempos hipermodernos. Barcelona: Editorial Anagrama; 2014: 13-35.

6. Weil S. Oppression and liberty. Taylor \& Francis Group; 1958: 79-80.

7. Gianinni H. Notas acerca de la moralidad de la acción. Revista de Filosofía 2011; 67: 167-182.

8. Millas J. Fundamentos de los derechos humanos. Revista Análisis 1982; VI(52): 35-37.

9. Giannini H. La metafisica eres tú. Santiago de Chile; Editorial Catalonia; 2007: 3-14.

10. De Chardin Th. El Fenómeno Humano. Editorial Taurus; 1967.

11. Monod J. El azar y la necesidad. Ensayo sobre la filosofía natural de la biología moderna. Tusquets editores; 2016: 17-33.

12. Valenzuela C. Aproximación ética científica al doble efecto o único bien posible en el aborto terapéutico. Acta Bioethica 2016; 22(2): 179-185.

13. Maturana Romesin H. La objetividad, un argumento para obligar. JC Sáez Editor; 1993: 151-156.

14. Bachelard G. La filosofía del no. Ensayo de una filosofía del nuevo espíritu cientifico. Amorrortu editores; 2009: 9-20.

15. Suzuki DT, Fromm E. Budismo Zen y Psicoanálisis. Fondo de Cultura Económica; 1964: 16.

16. Han B-Ch. La sociedad de la transparencia. Madrid: Editorial Herder, 1a edición; 2013: 10-30.

17. Calvino I. Seis propuestas para el próximo milenio. Ediciones Siruela; 2014: 85.

18. Benjamin W. La obra de arte en la época de su reproductibilidad técnica. Editorial Itaca, 1a edición; 2003: 79-94.

19. Lutereau L. El Objeto A como mirada: La "función cuadro". IV Congreso internacional de investigación y práctica profesional en Psicología. XIX Jornadas de Investigación. VIII Encuentro de Investigadores en Psicología del MERCOSUR. Buenos Aires: Facultad de Psicología. Universidad de Buenos Aires; 2012: 450-453. Disponible en https://www.aacademica.org/000-072/828.

20. Muñoz E. De la vulnerabilidad. La inquietud de la ética en Emmanuel Levinas. Santiago de Chile: Ril Editores; 2016 : 145-156.

21. Han B-Ch. Topología de la violencia. Madrid: Editorial Herder, $1^{\text {a }}$ edición; 2016: 41- 62.

22. Figueroa M. Filosofía y violencia en Jorge Millas. Revista de Filosofía 2011; 67: 145-165.

23. Augé M. Travesías y ficciones de la antropología en el contexto de la globalización. El objeto de la antropología hoy. Santiago de Chile: Pehuén; 2017: 23-36.

24. Foucault M. El nacimiento de la clínica, una arqueología de la mirada médica. Siglo Veintiuno Editores; $2008: 18$.

25. Schwember F. Dignidad, propiedad y necesidad en Robert Nozick. Santiago de Chile: Ril editores; 2016: 47-72.

Recibido: 18 de marzo de 2019

Aceptado: 10 de abril de 2019 